\title{
Review on the Application of Molecular Biotechnology for Crop Improvement
}

\author{
Dejen Bekis* \\ Ethiopian Institute of Agricultural Research, Fogera National Rice Research and Training Center (FNRRTC),
}

(Woreta) Bahir Dar, Ethiopia

*Corresponding Author: Dejen Bekis, Ethiopian Institute of Agricultural Research, Fogera National Rice Research and Training Center (FNRRTC), (Woreta) Bahir Dar, Ethiopia.

\begin{abstract}
Conventional breeding has increased food production and created thousands of today's crop varieties to meet a highly diversified demand of agricultural products. However, the recombination of genetic elements through mating and selection is not precise, the new developed crop through conventional breeding contain some undesirable genes. This affects the effectiveness of the breeding process. Besides, the success of the breeding process may be limited by the lack of germplasm with the desired traits. Hence, the aim of this paper is to review the recent achievement of genetic engineering/molecular biotechnology on crop improvement. Genetic engineering allows gene transfer between unrelated genera or species. Genetically engineered varieties that developed using recombinant DNA techniques in addition to sexual crossing and selection has created additional issues for seed genetic purity for producers seeking to meet organic marketing standards. Genetic engineering is very essential to improve crop production by modification of gene of interest via considering insect resistant, weed control, disease resistance, improved botanical pesticide, regulated gene expression, recombinant DNA technologies, gene tagging, gene mapping, gene sequence and function, analysis of metabolic pathways, genetic transformation, tolerance to abiotic stresses, sugar and starch metabolism, altering senescence, photosynthetic efficiency, yield and nutritional value. However, it needs a great effort to develop genetically modified high yielding crop varieties with the required quality.
\end{abstract}

Keywords: Conventional breeding, Crop improvement, Gene, Genetic engineering, Molecular Biotechnology

\section{INTRODUCTION}

Crop improvement through conventional breeding has dramatically increased food production and created thousands of today's crop varieties to meet a highly diversified demand of agricultural products. In conventional breeding, the genes preexisting within a species or its close relatives are brought together by sexual crossing and plants with desired characteristics are then selected. Because the recombination of genetic elements through mating and selection is not precise, the new developed crop, more or less, will contain some undesirable genes. This affects the effectiveness of the breeding process. Besides, the success of the breeding process may be limited by the lack of germplasm with the desired traits.

Genetic engineering is a DNA recombination technique that allows gene transfer between unrelated genera or species. This technique also has been used in crop improvement.

Compared with the conventional breeding, genetic engineering has its advantages. Firstly, it extends the genetic base because theoretically genetic engineering can transfer agronomical useful genes from any organism. Secondly, it is more effective because it avoids the problem of linkage drag associated with the conventional cross breeding and it is less time consuming. To date, many genetic engineered crops have been developed and commercialized (HuixiaShou, 2003).

Genetically engineered (GE) varieties (those developed using recombinant DNA techniques in addition to sexual crossing and selection) has created additional issues for seed genetic purity, particularly for producers seeking to meet organic marketing standards or who are engaged in international trade (Kent. 2006). This paper explains the application of genetic engineering/molecular biotechnology on crop improvement 


\section{Application of Genetic Engineering/Molecular Biotechnology in Crop IMPROVEMENT}

\subsection{Insect-Resistant}

Tolerance to damaging insect pests is another agronomic trait where there is a good fit with plant genetic engineering technology. Insect-resistant crops contain genes from the soil bacterium Bacillus thuringiensis $(\mathrm{Bt})$. The protein produced in the plant by the Bt gene is toxic to a targeted group of insectsfor example European corn borer or corn rootworm-but not to mammals. Transgenic crops are now grown in over 12 countries in the world. Successful control of cotton bollworms has been achieved through transgenic cotton. Cry type toxins from Bt are effective against cotton bollworm, corn earworm, the European corn borer, and rice stem borers. Successful expression of Bt genes against the lepidopterous pests has also been achieved in tomato, potato, brinjal, groundnut, and chickpea (Fischhoff, 1988; Gasser and Fraley,1989; Sharma et al., 2002).

\subsection{Weed Control}

Engineering herbicide tolerance into crops represents a new alternative for conferring selectivity and enhancing crop safety of herbicides. Research has largely concentrated on those herbicides with properties such as high unit activity, low toxicity, low soil mobility, and rapid biodegradation and with broad spectrum activity against various weeds. The development of crop plants that are tolerant to such herbicides would provide more effective, less costly, and more environmentally attractive weed control (Gasser and Fraley, 1989).

Two general approaches have been taken in engineering herbicide tolerance: (i) altering the level and sensitivity of the target enzyme for the herbicide and (ii) incorporating a gene that will detoxify the herbicide. As an example of the first approach, glyphosate, the active ingredient of Roundup herbicide, acts by specifically inhibiting the enzyme 5-enolpyruvylshikimate-3-phosphate synthase (EPSPS). Glyphosate is active against annual and perennial broadleaf and grassy weeds, has very low animal toxicity, and is rapidly inactivated and degraded in all soils. Tolerance to glyphosate has been engineered into various crops by introducing genetic constructions for the overproduction of EPSPS or of glyphosate-tolerant variant EPSPS enzymes. Similarly, resistance to sulfonylurea compounds, the active ingredients in Glean and Oust herbicides, has been produced by the introduction of mutant acetolactatesynthase (ALS) genes. Glean and Oust are broad-spectrum herbicides and are effective at low application rates. Resistance to gluphosinate and bromoxynil has been achieved by the alternative approach of introducing bacterial genes encoding enzymes that inactivate the herbicides by acetylation or nitryl hydrolysis, respectively (Gasser and Fraley, 1989).

\subsection{Disease Resistance}

There are many viruses, fungi and bacteria that cause plant diseases. Plant biologists are working to create plants with genetically-engineered resistance to these diseases through Antisense RNA mediated protection and coat protein-mediated protection were significant resistance to tobacco mosaic virus (TMV) infection, termed "coat protein-mediated protection," has been achieved by expressing only the coat protein gene of TMV in transgenic plants. This approach produced similar results in transgenic tomato, tobacco, and potato plants against a broad spectrum of plant viruses, including alfalfa mosaic virus, cucumber-mosaic virus, potato virus $\mathrm{X}$, and potato virus $\mathrm{Y}$. One mechanism of coat proteinmediated cross protection appears to involve interference with the un-coating of virus particles in cells before (Gasser and Fraley, 1989; Sharma et al., 2002).

Agrobacterium radiobacter was released commercially to control crowen gall (Agrobacterium tumefaciens) disease of stone fruit trees (Maurizio and David, 1996). Agrobacterium rhizogenes; Natural mechanisms of gene transfer to plant cells also operate in the induction of hairy root disease by Agrobacterium rhizogenes. Virulent strains of A. rhizogenes carry a Ri (root inducing) plasmid that shares key characteristics with Ti plasmids: A T-DNA and a virulence region. The virulence genes of $\mathrm{Ri}$ and Ti plasmids are very similar and can be interchanged (Newbury, 2003).

\subsection{Pollen Sterility}

Pollen-mediated transfer is widely believed to be the major contributor to gene flow in flowering plants. Interfering with the development of male reproductive structures through genetic engineering (GE) has been widely used as an effective strategy for production of male sterility in plants. These methods are 
distinctly different from cytoplasmic male sterility (CMS) and shown to be extremely effective and stable. The tapetum is the innermost layer of the anther wall that surrounds the pollen sac and is essential for the successful development of pollen. It has been shown that the tapetum produces a number of highly expressed messenger RNAs. Genes expressed exclusively in the anther are most likely to include those that control male fertility (Kauschet al., 2012).

\subsection{Improved Botanical Pesticide}

Only limited quantities of botanical pesticides are now used in developed countries in place of some synthetic pesticides. However, in some developing countries, including China and India, botanical pesticides such as neem were effectively used in creating the effectiveness of neem and other available botanical pesticides by genetic engineering would be an asset to farmers because they were relatively effective and safe (Maurizio and David, 1996).

\subsection{Regulated Gene Expression}

Genes that showed precise temporal and spatial regulation in leave, floral organs, seeds, and other plant organs have now been identified and isolated from a number of species of higher plants. In addition, a number of genes that respond to external influences, such as heat shock, an-aerobiosis, wounding, nutrients, and applied phytohormones, have been isolated and characterized. The control regions of these genes may also find utility in genetic engineering strategies. The ability to decrease the expression of a gene in a transgenic plant also has potential utility in the study of plant gene expression and function as well as in crop improvement. Significant successes have already been achieved with genes that produce antisense RNAs to the messengers for polygalacturonase in tomato fruits and chalcone synthase in petunia and tobacco plants (Gasser and Fraley, 1989)

\subsection{Recombinant DNA Technologies}

Besides generating information on gene sequences and function, allows the identification of specific chromosomal regions carrying genes contributing to traits of economic interest. The theoretical advantages of indirect selection using genetic markers were first reported by Sax nearly 80 years ago. However, it was not until the development of DNA marker technology that a sufficiently large number of genetic markers could be generated to accommodate the needs of modern plant breeding programs. There is now a profusion of different types of DNA markers, each having a differential set of conditions. The identification of DNA markers for traits of interest usually depends on making crosses between two genotypes with substantial and heritable differences in trait(s) of interest. Depending on the crop and traits involved, mapping populations are then derived from the progeny of this cross by selfing once, many times (recombinant inbred lines-RIL), back-crossing to one of the parental genotypes (BC) or plants subjected to tissue culture to generate double haploids (DH). A major advantage of RIL and DH mapping populations is that each line is homozygous and can, therefore, be eternally multiplied through self-pollination. This then allows the population to be evaluated under many environments and seasons, facilitating a much more accurate estimate of phenotypic variation on which to base the mapping exercise (Sharma et al., 2002).

\subsection{Gene Tagging}

Advances in methods for the identification and isolation of new gene coding sequences have a great importance to the engineering of improved plants. The cloning of transposon sequences has allowed the isolation of genes from several species by transposon-mediated gene tagging. The demonstration that mobile elements isolated from maize were able to transpose when introduced into dicot species indicates that powerful technique was applicable to any plant species for which transformation is possible. It has also been shown that under appropriate transformation conditions, the T-DNA of a plant transformation vector can itself serve as an insertional mutagen (Gasser and Fraley, 1989).

\subsection{Gene Mapping}

Major efforts have been mounted to obtain higher solution restriction fragment length polymorphism (RFLP) genetic maps in a number of plant species. The availability of such a map in tomato has already led to the resolution of several loci affecting quantitative and qualitative traits. The RFLP mapping technique has a powerful in Arabidopsis, here the small genome size and lack of significant repetitive sequences could simplify the process of genome "walking" from an RFLP marker to a closely linked gene. The availability of Arabidopsigsenomic libraries in cosmids, which can also act as plant 
transformation plasmids, that allow direct testing of the isolated DNA for its ability to complement the mutation of interest at each step of the walking process. In addition, such libraries may be used in largescale transformation experiments to directly rescue genes by complementing mutants with a selectable phenotype (Gasser and Fraley, 1989).

\subsubsection{QTL mapping}

QTL mapping relies on statistical linkage analyses among quantitative traits of interest and genetic markers, using a population that carries genetic mosaics derived from parental varieties, such as second generation (F2) plants or recombinant inbred lines (RILs) (Shin and Makoto,2008).

\subsubsection{Association Mapping}

Association mapping, also called linkage disequilibrium (LD) mapping, relies on correlation between a genetic marker and a phenotype among collections of diverse germplasm. Association scanning is performed by comparing phenotypic scores respective to each haplotype. Data sets are analyzed using statistical methods, which have been designed to deal with the population structure (Shin and Makoto,2008).

\subsection{Gene Sequence and Function}

Genes can be discovered using a variety of approaches, but a routine large-scale approach can commonly be followed by generating and sequencing a library of expressed genes. This library typically consists of thousands of strands of complementary DNA (cDNA) that are abundantly expressed by that plant under the given environmental conditions at the sampled growth stage. When sequenced, these cDNAs are termed expressed sequence tags (EST). A large number of ESTs are now available in the public databases for several model plants and crops such as rice, maize, sorghum, and soybean. A comparison of the EST databases from different plants can reveal the diversity in coding sequences between closely and distantly related plants, while mapping of ESTs may elucidate the synteny between those species. When a high level of sequence similarity is detected between an EST and a gene of known function in another species, it is possible to infer probable gene function in the species of interest. However, the emphatic elucidation of gene function still requires experimental verification. Only a small proportion of genes are abundantly transcribed in any particular environment or growth stage, and therefore, a complete picture can only be obtained by generating a range of cDNA libraries from plants grown under different environmental conditions and sampled at different growth stages or by sequencing entire cDNA genome library. For understanding gene functions of a whole organism, functional genomics technology is now focused on high throughput (HTP) methods using insertion mutant isolation, gene chips or microarrays, and proteomics. Finally, the identified genes are expressed in transgenic plants. These techniques offer powerful new uses for the genes discovered through sequencing (Sharma et al., 2002).

\subsection{Analysis of Metabolic Pathways}

Knowledge of the changes in a specific plant function induced by different treatments has led to the development of methods to isolate genes involved in the metabolic pathways or their associated physiology. With the availability of tagged mutant populations, the use of elegant screening systems based on knowledge of metabolism provides a relatively easy approach to isolating the genes for key steps. Many secondary plant metabolites such as flavonoids have been implicated in several functions in plant physiology, including host plant resistance to biotic stress factors. Many compounds of the flavonoid biosynthetic pathway (flavanones, flavones, flavanols and isoflavonoids) accumulate in response to biotic and abiotic stresses (Sharma et al., 2002).

\subsection{Genetic Transformation}

Genetic transformation offers direct access to a vast pool of useful genes not previously accessible to plant breeders. Current genetic engineering techniques allow the simultaneous use of several desirable genes in a single event, thus allowing coordinated approaches to the introduction of novel genes/traits into the elite background. Genetic engineering also offers the possibility of introducing a desirable character from closely-related plants without associated deleterious genes or from related species, which do not readily cross with the crop of interest or from completely unrelated species even in other taxonomic phyla (Sharma et al., 2002). 


\subsection{Tolerance to Abiotic Stresses}

Development of crops with an inbuilt capacity to withstand abiotic stresses would help stabilize the crop production and significantly contribute to food security in developing countries. In bacteria, trehalose is produced by the action of trehalose phosphate synthase, which produces trehalose phosphate and trehalose phosphate phosphatase-which degrades trehalose-6- phosphate into trehalose. When these two enzymes are expressed in transgenic plants, the plants have larger leaves, altered stem growth, and improved response to stress. Over-expression of various glutamate dehydrogenases (GDH) also improves plant growth and stress tolerance. Plants have been specifically transformed with genes encoding a-and b-subunits of the chloroplast-located GDH from the alga, Chlorella sorokiniana. Similar improvements in performance have been reported for rice plants transformed with the barley late embryogenesis (LEA) gene. Plants with an ability to produce more citric acid in roots provide tolerance to aluminium in acid soils. Introduction of functional calcineurin activity provides tolerance to salinity involving the introduction of a gene encoding a plant farnesyltransferase and inhibitors of this enzyme when expressed in plants, enhance drought tolerance, delay senescence, and modify the growth habit. A salt tolerance gene isolated from man groove (Avicennia marina) has been cloned, and can be transferred into other crop plants the gutD gene from Escherichia coli can also be used to provide salt tolerance. These genes hold a great potential for increasing crop production in marginal lands (Sharma et al., 2002). Drought tolerance is enhanced by delaying leaf senescence when an isopentenyl transferase gene for cytokinin synthesis is expressed under the control of a stress and maturation induced promoter (Takeda and Matsuoka, 2008).

\subsection{Sugar and Starch Metabolism}

Sucrose phosphate synthase (SPS) is a key enzyme in the regulation of sucrose metabolism. Transgenic plants expressing the maize SPS under the control of a promoter from the small subunit of tobacco. Rubisco have shown increased foliar sucrose/starch ratios in leaves, and decreased amounts of foliar carbohydrates when grown with $\mathrm{CO} 2$ enrichment. Modification of the activity of metabolites of the TCA (tricarboxylic acid) cycle by reducing the amount of the NAD-malic enzyme can also be used for increasing starch concentrations. Introduction of the Escherichia coli inorganic pyrophosphatase to alter the amount of sugar, and modification of hexokinases, which affect the sugar-sensing capacities of a plant as well as sucrose binding proteins and a class of cupin protein have been implicated in sugar unloading in developing legume seeds. This has opened up exciting possibilities for changing the chemical composition of food grains to meet specific requirements (Sharma et al., 2002).

\subsection{Altering Senescence}

Leaf senescence leads to a progressive death of the leaf or a plant upon aging due to reduction in the production in cytokinin. Cytokinin is a plant hormone that naturally prevents senescence and maintains photosynthetic activity in leaves. Reduction in leaf senescence would improve the performance of a plant, and thereby increase the crop yield. This in part can be achieved through stay green trait in maize, sorghum, pearl millet, and other cereal crop. Stay green trait in sorghum is also associated with adaptation to drought stress. Introduction of farnesyltransferase and isopentenyl transferase (IPT) genes delays senescence. The process of leaf senescence can be blocked through a gene encoding the cytokinin-synthesis enzyme, isopentenyl transferase. When transformed with the SAG12-IPT construct, a plant can produce enough cytokinin to delay leaf senescence. Cytokinin production is triggered only at the onset of senescence, due to regulation of the IPT gene by the senescence-specific SAG12 promoter. Thus, the plant grows normally without an excess of cytokinin until hormone is needed to block senescence. This avoids problems with unregulated, constitutive over-production of cytokinin, such as short, bushy plants, and decreased root growth. Commercial uses for delayed senescence include increasing plant vegetative growth, seed and fruit production, prolonging the shelf-life of vegetables, maintaining nitrogen content of forage crops (e.g. alfalfa), provide a safe and natural source of cytokinin, and produce transgenic plants of multiple species. The promoter could also be combined with other genes, whose targeted expression during senescence would be beneficial

\subsection{Photosynthetic Efficiency and Improved Yield}

An exciting experimental approach to increase crop yield radically is to change components of plant biochemistry with respect to introducing the $\mathrm{C} 4$ type of photosynthesis into a $\mathrm{C} 3$ plants such as Arabidopsis and potato. $\mathrm{C} 3$ photosynthesis suffers from $\mathrm{O} 2$ inhibition due to the oxygenase reaction of 
ribulose 1, 5- biophosphate carboxylase/oxygenase (Rubisco), and the subsequent loss of $\mathrm{CO}_{2}$ from photorespiration. In contrast, $\mathrm{C} 4$ plants such as maize have evolved a biochemical mechanism to overcome this inhibition. A key feature of this mechanism is the activity of phosphoenolpyruvate carboxylase (PEPC), an enzyme that fixes atmospheric $\mathrm{CO}_{2}$ in the cytosol of mesophyll cells. Using an Agrobacterium-mediated transformation system, the intact maize PEPC has recently been transferred into the $\mathrm{C} 3$ plants. Physiologically, these plants exhibited reduced $\mathrm{O}_{2}$ inhibition of photosynthesis and had photosynthetic rates comparable to those of control untransformed plants. Investigations into the manipulation of the key photosynthetic enzymes, Rubisco, pyruvate phosphate kinase(PPDK), and NADP malate dehydrogenase (NADPMDH) in the $\mathrm{C} 4$ dicotyledonous species Flaveriabidentishave also been reported. An alternative strategy to reduce photorespiration by manipulating catalase amounts in tobacco has also been described.

Appropriate manipulation of the enzymes involved in photosynthetic activity can be used to increase the productivity potential of $\mathrm{C} 3$ plants. Genes determining plant height in Arabidopsis are orthologous (similar) to dwarf genes in cereals, which have been used in conventional plant breeding in the 'Green Revolution'. These genes (NORIN 10) were introduced into western wheat varieties in the 1950s, and have now been isolated, and identical phenotypes reconstructed in other crops through genetic transformation. These dwarfing genes can now be routinely deployed in various crop species to increase crop productivity. Improved yield can also be achieved by manipulation of fructose-1,6-bisphosphate aldolase(FDA), an enzyme that reversibly catalyses the conversion of triosephosphate to fructose-1,6bisphosphate. Leaves of transgenic plants expressing FDA from E. coli in the chloroplast show significantly enhanced starch accumulation, lower sucrose concentration, and higher root mass. A more generic method for changing plant performance may be to modify plastid number, and the expression of a hybrid protein comprising a yeast gene encoding 5 -amino levulinic acid synthase and an $\mathrm{N}$-terminal transit sequence for the small subunit of carboxydismutase. Manipulation of chlorophyll a/b binding genes has also been used to modify chlorophyll amounts. Degreening of oilseed rape caused by sublethal freezing during seed maturation can be accomplished by anti-sense reduction of the type I chlorophyll $\mathrm{a} / \mathrm{b}$ binding protein of light harvesting complex II. Other non-photosynthetic approaches to increasing yield of both shoot and root include over expression of a cyclin gene, such as cycla gene from Arabidopsis (Sharma et al., 2002).

\subsection{Nutritional Factors}

Several quality traits can be targeted to improve the nutritional status of crop produce. These include carbohydrates, proteins, oils, vitamins, iron, and amino acids. The selection of target traits is influenced by the end users, producers, and agro-based industry. Research in this area epitomizes the change in emphasis from single gene agronomic traits of herbicide and insect tolerance to more complex traits of direct benefit to the consumer such as modified seed quality. For example, transgenic rice, with a capacity to produce beta-carotene, can be used to overcome the deficiency of vitamin A. Similarly, transgenic rice with elevated levels of iron has been produced using genes involved in the production of an iron binding protein that facilitates iron availability in human diet. Altering protein levels, composition of fatty acids, vitamins and amino acids is being increasingly targeted for value addition. It is now possible to alter the composition of fatty acids so that polyunsaturated (e.g. linoleic acid) content is decreased while that of mono-unsaturated (e.g. oleic acid) content is increased to allow processing without the traditional use of hydrogenation, and thus avoiding the undesirable trans-fatty acids. Amounts of essential amino acids such as lysine, methionine, threonine, and tryptophan can be increased to improve the nutritional quality of cereal grains. Transgenic modifications have also been used to alter the ratio of amylose to amylopectin in starch. Decreasing the amounts of oligosaccharides (such as raffinose and stachyose) improves digestibility, and decreases the degree of flatulence during digestion. Transgenic technology can also be used to remove anti-nutritional factors (Sharma et al., 2002).

\section{CONCLUSION}

Rapidly increasing world population is depending on the continuous supply of agricultural product. To date, increment of food production is the most urgent issue. Recombination of genetic elements through mating and selection is not precise, the new developed crop, more or less, will contain some undesirable genes. This affects the effectiveness of the breeding process. Besides, the success of the breeding process may be limited by the lack of germplasm with the desired traits. Genetic engineering is a DNA recombination technique that allows gene transfer between unrelated genera or species. This technique 
can solve a lot challenges in crop improvement. Thus, insect resistant, weed control, disease resistance, improved botanical pesticide, regulated gene expression, recombinant DNA technologies, gene tagging, gene mapping, gene sequence and function, analysis of metabolic pathways, genetic transformation, tolerance to abiotic stresses, sugar and starch metabolism, altering senescence, photosynthetic efficiency and improved yield and nutritional factors are very crucial application on crop improvement.

\section{REFERENCES}

[1] Maurizio G. Paoletti and David Pimentel, 1996.Genetic engineering in Agriculture and the environment. Assessing risk and benefits.

[2] H.C. Sharma , J.H. Crouch, K.K. Sharma, N. Seetharama, C.T. Hash, 2002. Review on applications of biotechnology for crop improvement: prospects and constraints.

[3] Charles S. Gasser and Robert T. Fraley, 1989. Genetically Engineering Plants for Crop Improvement.

[4] HuixiaShou, 2003. Crop improvement through genetic engineering: development of transformation technologies and production of stress tolerant transgenic crops.

[5] Shin Takeda and Makoto Matsuoka, 2008. Genetic approaches to crop improvement: responding to environmental and population changes.

[6] David A. Fischhoff, 1988.Application of genetic engineering to crop protection.

[7] Kent J. Bradford. 2006. Methods to maintain genetic purity of seed stocks.

[8] H. John Newbury, 2003. Plant Molecular Breeding.

[9] Albert P. Kausch, Joel P. Hague 1, Stephen L. Dellaporta 2, Maria A. Moreno 2, Chip Longo 1, Kimberly Nelson.2012. Pollen Sterility-A Promising Approach to Gene Confinement and Breeding for Genetically Modified Bioenergy Crops. Journal of Agriculture,2:295-315; doi:10.3390/agriculture2040295

Citation: Dejen Bekis," Review on the Application of Molecular Biotechnology for Crop Improvement" International Journal of Research Studies in Biosciences (IJRSB), 8(1), pp 28-34. DOI: http://dx.doi.org/10.20431/2349-0365.0801004

Copyright: (ㅇ 2020 Authors. This is an open-access article distributed under the terms of the Creative Commons Attribution License, which permits unrestricted use, distribution, and reproduction in any medium, provided the original author and source are credited. 